\title{
金属钠催化下一元酯的高效无溶剂胺化反应
}

\author{
沈 涛 ${ }^{a}$ 欧阳博 $a, b$ 周少东 ${ }^{a}$ 钱 超*, ${ }^{*}$ 陈新志 ${ }^{a}$ \\ ( ${ }^{a}$ 浙江大学浙江省化工高效制造技术重点实验室 杭州 310027)

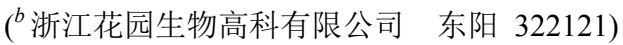

\begin{abstract}
摘要 开发了一种金属钠催化下一元酯的高效无溶剂胺化反应方法. 通过金属钠与氨基钠对一元酯的胺化反应的对比 实验，发现金属钠和液氨原位生成的氨基负离子对反应活性更高，提出了可能的反应机理. 该方法反应速度快，反应 条件温和, 适合多种一元酯的胺化.

关键词 一元酯; 胺化; 钠; 氨基钠
\end{abstract}

\section{Efficient, Solvent-Free Aminolysis of Monoesters Catalyzed by Sodium}

\author{
Shen, Tao ${ }^{a} \quad$ Ouyang, Bo ${ }^{a, b} \quad$ Zhou, Shaodong $^{a} \quad$ Qian, Chao ${ }^{*, a} \quad$ Chen, Xinzhi ${ }^{a}$ \\ ( ${ }^{a}$ Zhejiang Provincial Key Laboratory of Advanced Chemical Engineering Manufacture Technology, \\ Zhejiang University, Hangzhou 310027) \\ ( ${ }^{b}$ Zhejiang Garden Biochemical High-Tech Co., LTD, Dongyang 322121)
}

\begin{abstract}
An efficient, solvent-free procedure using sodium as catalyst for the aminolysis of monoesters is reported. A detailed comparison of catalysts between sodium and sodium amide was made. It was found that the fresh sodium amide by in-situ synthesis of sodium with ammonia was more active than the indirectly adding sodium amide. As compared to the previously reported approaches, the procedure given in this work is much faster and performed under mild conditions. Furthermore, this procedure is applied successfully for the aminolysis of other monoesters.

Keywords monoester; aminolysis; sodium; sodium amide
\end{abstract}

\section{Introduction}

Amide constitutes one of the most important function units in various compounds. ${ }^{[1,2]}$ In recent years, considerable efforts have been devoted to economic and eco-friendly synthesis, ${ }^{[3]}$ especially solvent free method. ${ }^{[4]}$ However, the efficiency of amide-bond formation is always hampered by poor atom economy and the limited scope of substrates. ${ }^{[5]}$ Ester aminolysis is the classical method for making amides, regardless of the lower level activation of esters than acyl halides, anhydrides acyl azides and acylimidazoles, as esters produce fewer side reactions. ${ }^{[6]}$ For example, acyl chlorides $^{[7]}$ were prone to be hydrolyzed in the reaction with ammonia, the same holds true for the reaction of trifluoroacetic anhydride ${ }^{[8]}$ with ammonia in methanol. Carboxylic acid ammoniolysis is also a common method for making amides. Carboxylic acids ${ }^{[9]}$ undergo neutralization and then dehydration to produce the amides. However, harsh conditions like microwave are always required due to the poor reactivity of carboxylic acids. ${ }^{[10]}$ In addition, nitrile, ${ }^{[11,12]}$ aldehyde, ${ }^{[13,14]}$ alcohol ${ }^{[15,16]}$ and alkyne ${ }^{[17]}$ were also employed in the conversion to amides. Cyanide anion was found to be an efficient catalyst for the aminolysis of substituted phenyl esters in alcoholic solution, ${ }^{[18]}$ and aromatic carboxylic esters are converted to secondary amides using $\mathrm{Zn}$ dust as catalyst when microwave is subjected in the presence of $N, N$-dimethylformamide. Conventional heating is also valid if tetrahydrofuran is used as solvent. ${ }^{[19]}$ By contrast, aminolysis of alkyl ester is always achieved with longer reaction time and lower yield. For example, it costs $18 \mathrm{~h}$ for methyl 2-(methylthio)acetate to be converted to 2-(methylthio)acetamide at room tempera-

\footnotetext{
* Corresponding author. E-mail: qianchao@zju.edu.cn

Received August 7, 2018; revised September 28, 2018; published online October 26, 2018.

Project supported by the National Natural Science Foundation of China (No. 21476194) and the National Key Research and Development Program of China (No. 2016YFB0301800).
}

国家自然科学基金(No. 21476194)和国家重点研发计划(No. 2016YFB0301800)资助项目 
ture in the presence of ammonia. ${ }^{[20]}$ This number amounts to $15 \mathrm{~h}$ for methyl-acetate conversion at $180{ }^{\circ} \mathrm{C}$ in the presence of 2-(2-(aminomethoxy)ethoxy)ethanamine. ${ }^{[21]}$ $N$-Butyllithium, Grignard reagent and sodium amide were reported to be capable of enhancing the aminolysis of simple alkyl esters. ${ }^{[22]}$ Free radical reaction ${ }^{[23,24]}$ and Passerini reaction ${ }^{[25]}$ were also reported to synthesize amide.

Herein, we report that sodium is an ideal catalyst in the solvent-free ammonolysis of ethyl acetate. The applicability of this approach was also tested for various monoesters, and the corresponding products were obtained with $>95 \%$ yield under mild conditions.

\section{Results and discussion}

The aminolysis of ethyl acetate was selected as the model reaction. As shown in Table 1, the aminolysis did occur though rather slowly at $90{ }^{\circ} \mathrm{C}$ (Table 1, Entries 1,2) and the yield could be improved further with temperature increasing (Table 1, Entries 3 6). However, the yield only reached up to $71.5 \%$, even prolonging reaction time to $10 \mathrm{~h}$ (Table 1, Entry 6). In order to improve the yield and reduce reaction time, various base catalysts were screened. As shown in Table 2, when sodium was employed, the product was obtained in $98.8 \%$ yield at $110{ }^{\circ} \mathrm{C}$ (Table 2, Entry 11). When sodium amide was employed, the products were obtained in 96\% 97\% (Table 2, Entries 13 15), and when sodium carbonate, calcium oxide and sodium methoxide were employed, the product were obtained in $35 \%$ 55\% (Table 2, Entries 3, 6, 9). The results demonstrated that sodium and sodium amide promoted the reaction while sodium carbonate, calcium oxide and sodium methoxide all failed. The amount of sodium was then evaluated. Figure 1 shows that the optimum amount of sodium to be used was 0.06 equiv. while a lower ratio worked worse significantly and a higher ratio did not further function.

Table 1 Aminolysis of ethyl acetate without catalyst ${ }^{a}$

\begin{tabular}{|c|c|c|c|}
\hline Entry & Temperature $/{ }^{\circ} \mathrm{C}$ & Time/h & Yield $^{b} / \%$ \\
\hline 1 & 90 & 7 & 1.1 \\
\hline 2 & 90 & 10 & 6.8 \\
\hline 3 & 140 & 7 & 15.3 \\
\hline 4 & 140 & 10 & 26.6 \\
\hline 5 & 170 & 7 & 60.4 \\
\hline 6 & 170 & 10 & 71.5 \\
\hline
\end{tabular}

\footnotetext{
${ }^{a}$ Reaction conditions: ethyl acetate $(0.3 \mathrm{~mol})$, ammonia $(1.2 \mathrm{~mol}) .{ }^{b} \mathrm{GC}$ yield.
}

The proposed mechanism for aminolysis of ethyl acetate was presented in Figure 2. The concentration of amide anions was a key factor affecting the rate-determining step in this reaction. When the reaction was employed without catalyst (a), amide anions were obtained only by molecular autoionisation of ammonia. When the sodium amide was

Table 2 Aminolysis of ethyl acetate with different catalysts ${ }^{a}$

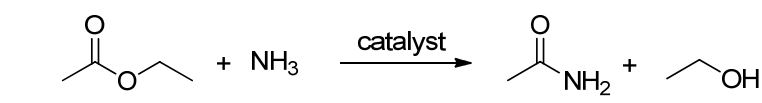

\begin{tabular}{clcc}
\hline Entry & Catalyst (0.1 equiv.) & Temperature $/{ }^{\circ} \mathrm{C}$ & Yield $^{b} / \%$ \\
\hline 1 & Sodium carbonate & 90 & 7.4 \\
2 & Sodium carbonate & 110 & 12.6 \\
3 & Sodium carbonate & 140 & 35.4 \\
4 & Calcium oxide & 90 & 10.3 \\
5 & Calcium oxide & 110 & 17.8 \\
6 & Calcium oxide & 140 & 45.5 \\
7 & Sodium methoxide & 90 & 15.3 \\
8 & Sodium methoxide & 110 & 36.8 \\
9 & Sodium methoxide & 140 & 55.5 \\
10 & Sodium & 90 & 98.7 \\
11 & Sodium & 110 & 98.8 \\
12 & Sodium & 140 & 98.7 \\
13 & Sodium amide & 90 & 96.8 \\
14 & Sodium amide & 110 & 97.2 \\
15 & Sodium amide & 140 & 97.1 \\
\hline
\end{tabular}

${ }^{a}$ Reaction conditions: ethyl acetate $(0.3 \mathrm{~mol})$, ammonia $(1.2 \mathrm{~mol})$, catalyst (0.03 mol), 7 h. ${ }^{b} \mathrm{GC}$ yield.

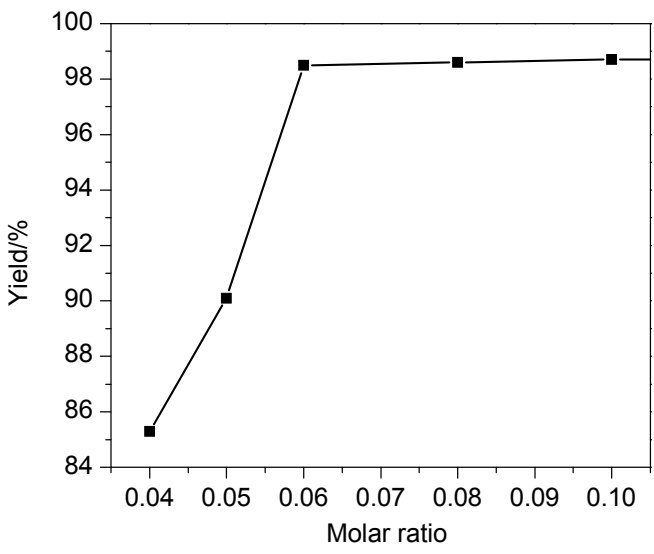

Figure 1 Effects of sodium amount on the aminolysis of ethyl acetate

Reaction conditions: $90{ }^{\circ} \mathrm{C}, \mathrm{NH}_{3}$ (4 equiv.), $7 \mathrm{~h}, \mathrm{GC}$ yield.

(a)

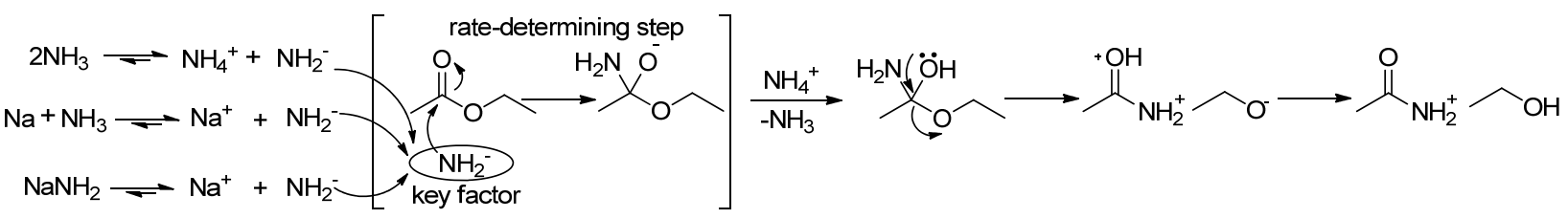

Figure 2 Proposed mechanism for aminolysis of ethyl acetate and three main sources of amide anions 
employed (c), amide anions were mainly obtained by ionization of sodium amide, which increased the concentration of amide anions and then promoted the whole reaction. And when the sodium was employed (b), sodium reacted with ammonia to produce sodium amide. ${ }^{[26]}$ This fresh and in-situ synthesized sodium amide increased the concentration of amide anions directly without ionization process. It was more important that this kind of amide anions were more active.

In order to verify the mechanism, sodium amide was used as a catalyst in the similar reaction conditions (Table 2, Entries 13, 14, 15). As evident from these three high yields, sodium amide did promote this reaction. At the same time from the results of Figure 3, a significant comparative advantage using sodium as catalyst was identified.

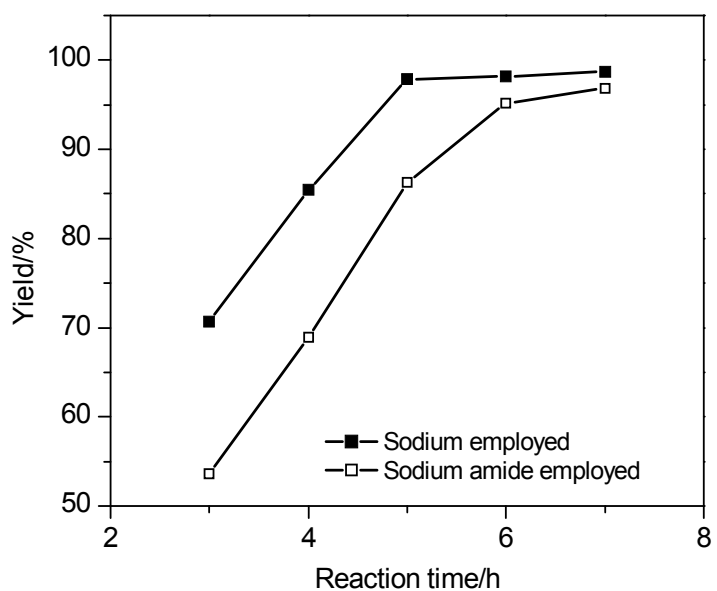

Figure 3 Effect of reaction time on the reactions employed with sodium or sodium amide

Reaction conditions: ammonia (4 equiv.); catalyst ( 0.06 equiv.), $90{ }^{\circ} \mathrm{C}$; GC yield.

Further, the aminolysis of various esters was examined, and the detailed results are shown in Table 3. In general, the aminolysis of all selected esters achieved with good to excellent yields $(84.8 \% \sim 96.5 \%)$. When the $\mathrm{R}^{1}$ group was an aliphatic group, methyl propionate gave its product in 96\% yield (Table 3, Entry 1), but methyl hexanoate only gave its product in $85.1 \%$ yield with higher temperature. As seen from the result (Table 3, Entries 1 4), longer carbon chain was involved, and harsher condition was required. When $\mathrm{R}^{1}$ or $\mathrm{R}^{2}$ group was a phenyl group, corresponding products were obtained in $96.5 \%, 84.8 \%, 96.2 \%$ and $92.6 \%$ yields, respectively (Table 3, Entries 5 8). The above reactions confirmed the wide substrate scope of this procedure in terms of aminolysis of monoesters under mild conditions.

\section{Conclusions}

In sum, an efficient, mild, and solvent-free procedure for the aminolysis of monoesters using sodium as catalyst has been presented. It has been demonstrated that the fresh sodium amide in-situ synthesized by sodium with ammonia was more active than the indirectly adding sodium amide by a comparative study. In addition, the mechanism for aminolysis of ethyl acetate was proposed. The procedure in this study is expanded extensively to other monoesters.

Table 3 Aminolysis of other esters using sodium ${ }^{a}$

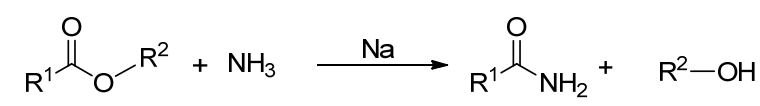

\begin{tabular}{|c|c|c|c|c|c|}
\hline Entry & Ester & $\begin{array}{c}\text { Ammonia/ } \\
\text { equiv. }\end{array}$ & Temp. $/{ }^{\circ} \mathrm{C}$ & Time/h & Yield $^{b} / \%$ \\
\hline 1 & & 3 & 90 & 6 & 96 \\
\hline 2 & & 4 & 120 & 6 & 87.5 \\
\hline 3 & & 4 & 120 & 8 & 85.3 \\
\hline 4 & & 4 & 145 & 6 & 85.1 \\
\hline 5 & & 5 & 140 & 5 & 96.5 \\
\hline 6 & & 5.5 & 140 & 5.5 & 84.8 \\
\hline 7 & & 5 & 140 & 4 & 96.2 \\
\hline 8 & & 5 & 140 & 5 & 92.6 \\
\hline
\end{tabular}

${ }^{a}$ Reaction conditions: methyl propionate $(0.3 \mathrm{~mol})$, methyl butyrate $(0.3 \mathrm{~mol})$, methyl isobutyrate $(0.3 \mathrm{~mol})$, methyl hexanoate $(0.3 \mathrm{~mol})$, methyl benzoate $(0.3 \mathrm{~mol})$, methyl 2-phenylacetate $(0.2 \mathrm{~mol})$, phenyl acetate $(0.2 \mathrm{~mol})$, benzyl acetate $(0.2 \mathrm{~mol})$, sodium $(0.06$ equiv. $) .{ }^{b} \mathrm{GC}$ yield.

\section{Experimental section}

\subsection{General procedure}

$N$-Propylamine, sodium methoxide, sodium, sodium amide and all the ester reagents were commercial products from Aladdin $^{\circledR}$ with the highest purity available $(\geqslant 97 \%)$ and were used without further purification. $N, N$-Dimethylformamide (DMF) and all inorganic reagents were commercial products from Sinopharm Chemical Reagent Co., Ltd. with a purity of over $99 \%$ and were used as received.

A mixture of ester reagent, basic catalyst (Tables $1 \sim 3$ ) were added to a $100 \mathrm{~mL}$ high pressure reaction kettle equipped with stirrer and a temperature measuring device. The kettle was purged with nitrogen gas until the pressure was $3.0 \mathrm{MPa}$ and kept steady for $30 \mathrm{~min}$ in order to prove that it was sealed well. And then the nitrogen gas was run- 
ning out by opening the outlet vale. It was repeated three times to replace all the air in the kettle. After this, certain amount of liquid ammonia (Table $1 \sim 3$ ) was added to the kettle from the inlet vale. The reaction mixture was heated slowly to the reaction temperature (Table $1 \sim 3$ ). The reaction was continued for its reaction time (Table $1 \sim 3$ ) with stirring at the reaction temperature. After completion of the reaction, the mixture was cooled to room temperature $\left(20{ }^{\circ} \mathrm{C}\right)$, and the rest ammonia was released into recycle system by opening outlet vale. These mixtures were evaporated to dryness under reduced pressure, and then the crude products were purified by recrystallization using ethanol. After filtration, the solvent was removed and the products were got. Gas chromatography (GC) detection was used after the products were dissolved in $50 \mathrm{~mL}$ of $N, N$-dimethylformamide.

\subsection{Product analysis}

GC analyses were performed on a GC Agilent $1790 \mathrm{~F}$ series. Quantitative analysis of the products was carried out by external standard method using GC analyses. Gas Chromatography-Mass Spectrometer (GC-MS) analyses were performed on a GC-MS Agilent 5973-6890 series (FID detector, weakly polar capillary column SE-30, nitrogen as carrier gas). ${ }^{1} \mathrm{H}$ NMR spectra were recorded in $\mathrm{CDCl}_{3}$ on a Bruker DRX 400 spectrometer. Melting points were determined in open capillaries using an Electrothermal 9100 without further corrections.

Acetamide: m.p. $80 \sim 81{ }^{\circ} \mathrm{C}$ (lit. ${ }^{[27]} 81.1{ }^{\circ} \mathrm{C}$ ); MS (EI, $70 \mathrm{eV}) \mathrm{m} / z(\%): 59\left(\mathrm{M}^{+}, 100\right), 44(80), 43$ (56); ${ }^{1} \mathrm{H}$ NMR $\left(400 \mathrm{MHz}, \mathrm{CDCl}_{3}\right) \delta: 6.13 \sim 5.80(\mathrm{~m}, 1 \mathrm{H}), 5.71(\mathrm{~s}, 1 \mathrm{H})$, 1.94 (s, 3H).

Propionamide: m.p. $79 \sim 80{ }^{\circ} \mathrm{C}$ (lit. ${ }^{[28]} 79{ }^{\circ} \mathrm{C}$ ); ${ }^{1} \mathrm{H}$ $\operatorname{NMR}\left(400 \mathrm{MHz}, \mathrm{CDCl}_{3}\right) \delta: 6.15(\mathrm{~s}, 1 \mathrm{H}), 5.72(\mathrm{~s}, 1 \mathrm{H}), 2.19$ (q, $J=7.60 \mathrm{~Hz}, 2 \mathrm{H}), 1.09$ (t, $J=7.60 \mathrm{~Hz}, 3 \mathrm{H})$.

Butyramide: m.p. $114 \sim 115{ }^{\circ} \mathrm{C}$ (lit. $\left.{ }^{[29]} 115 \sim 116{ }^{\circ} \mathrm{C}\right)$; ${ }^{1} \mathrm{H}$ NMR (400 MHz, $\left.\mathrm{CDCl}_{3}\right) \delta: 6.10(\mathrm{~s}, 1 \mathrm{H}), 5.65(\mathrm{~s}, 1 \mathrm{H})$, $2.13(\mathrm{t}, J=7.52 \mathrm{~Hz}, 2 \mathrm{H}), 1.80 \sim 1.24(\mathrm{~m}, 2 \mathrm{H}), 0.90(\mathrm{t}, J=$ $7.80 \mathrm{~Hz}, 3 \mathrm{H})$.

Isobutyramide: m.p. $127 \sim 128{ }^{\circ} \mathrm{C} \quad\left(\right.$ lit. $^{[30]} 126.5 \sim$ $\left.127{ }^{\circ} \mathrm{C}\right) ;{ }^{1} \mathrm{H} \mathrm{NMR}\left(400 \mathrm{MHz}, \mathrm{CDCl}_{3}\right) \delta: 5.94(\mathrm{~s}, 1 \mathrm{H}), 5.53$ (s, 1H), $2.36(\mathrm{dt}, J=13.80,6.9 \mathrm{~Hz}, 1 \mathrm{H}), 1.11(\mathrm{~d}, J=6.92$ $\mathrm{Hz}, 6 \mathrm{H})$.

Hexanamide: m.p. $100 \sim 101{ }^{\circ} \mathrm{C}$ (lit. ${ }^{[31]} 100{ }^{\circ} \mathrm{C}$ ); ${ }^{1} \mathrm{H}$ NMR (400 MHz, $\left.\mathrm{CDCl}_{3}\right) \delta: 6.09(\mathrm{~s}, 1 \mathrm{H}), 5.61(\mathrm{~s}, 1 \mathrm{H})$, $2.38 \sim 1.98(\mathrm{~m}, 2 \mathrm{H}), 1.66 \sim 1.45(\mathrm{~m}, 2 \mathrm{H}), 1.35 \sim 1.09(\mathrm{~m}$, $4 \mathrm{H}), 0.93 \sim 0.70(\mathrm{~m}, 3 \mathrm{H})$.

Benzamide: m.p. $130 \sim 131{ }^{\circ} \mathrm{C}$ (lit. ${ }^{[32]} 130 \sim 131{ }^{\circ} \mathrm{C}$ ); ${ }^{1} \mathrm{H}$ NMR $\left(400 \mathrm{MHz}, \mathrm{CDCl}_{3}\right) \delta: 7.75 \sim 7.33(\mathrm{~m}, 5 \mathrm{H}), 6.10$ (s, 1H), $6.02 \sim 5.80(\mathrm{~m}, 1 \mathrm{H})$.

Benzeneacetamide: m.p. $155 \sim 156{ }^{\circ} \mathrm{C}$ (lit. ${ }^{[33]} 157 \sim$ $\left.158{ }^{\circ} \mathrm{C}\right) ;{ }^{1} \mathrm{H}$ NMR $\left(400 \mathrm{MHz}, \mathrm{CDCl}_{3}\right) \delta: 7.44 \sim 7.20(\mathrm{~m}$, $5 \mathrm{H}), 5.65(\mathrm{~s}, 1 \mathrm{H}), 5.36(\mathrm{~s}, 1 \mathrm{H}), 3.52(\mathrm{~d}, J=3.20 \mathrm{~Hz}, 2 \mathrm{H})$.

Supporting Information Experimental details and proton nuclear magnetic resonance of synthesized products. The Supporting Information is available free of charge via the
Internet at http://sioc-journal.cn.

\section{References}

[1] Montalbetti, C. A. G. N.; Falque, V. Tetrahedron 2005, 61, 10827.

[2] Dong, H.; Hou, M. Chin. J. Org. Chem. 2017, 37, 267 (in Chinese). (董浩, 侯梅芳, 有机化学, 2017, 37, 267.)

[3] (a) Xie, L. Y.; Li, Y. J.; Qu, J.; Duan, Y.; Hu, J.; Liu, K. J.; Cao, Z.; He, W. M. Green Chem. 2017, 19, 5642.

(b) Xie, L. Y.; Qu, J.; Peng, S.; Liu, K. J.; Wang, Z.; Ding, M. H.; Wang, Y.; Cao, Z.; He, W. M. Green Chem. 2018, 20, 760

(c) Tan, J.; Guo, Y.; Zeng, F.; Chen, G.; Xie, L.; He, W. Chin. J. Org. Chem. 2018, 38, 1740 (in Chinese).

(谭家希，郭也，曾飞，陈观荣，谢龙勇，何卫民，有机化学， 2018, 38, 1740.)

(d) Xie, L. Y.; Peng, S.; Lu, L. H.; Hu, J.; Bao, W. H.; Zeng, F.; Tang, Z.; Xu, X.; He, W. M. ACS Sustainable Chem. Eng. 2018, 6 , 7989.

[4] (a) Zhang, Q.; Wang, X.; Xiao, Q.; Yin, D. Chin. J. Org. Chem. 2017, 37, 954 (in Chinese).

(张青扬, 汪小涧, 肖琼, 尹大力, 有机化学, 2017, 37, 954.)

(b) Liu, K. J.; Jiang, S.; Lu, L. H.; Tang, L. L.; Tang, S. S.; Tang, H. S.; Tang, Z.; He, W. M.; Xu, X. Green Chem. 2018, 20, 3038.

(c) Wu, C.; Lu, L. H.; Peng, A. Z.; Jia, G. K.; Peng, C.; Cao, Z.; Tang, Z.; He, W. M.; Xu, X. Green Chem. 2018, 20, 3683.

(d) Liu, T.; Peng, Y.; Wang, Y.; Yong, J.; Wang, X. Chin. J. Org. Chem. 2018, 38, 969 (in Chinese).

(刘天宝，彭艳芬，王雅洁，雍家远，汪新，有机化学，2018，38， 969.)

[5] Constable, D. J. C.; Dunn, P. J.; Hayler, J. D.; Humphrey, G. R.; Leazer, J. J. L.; Linderman, R. J.; Lorenz, K.; Manley, J.; Pearlman, B. A.; Wells, A.; Zaks, A.; Zhang, T. Y. Green Chem. 2007, 9, 411.

[6] Joullie, M. M.; Lassen, K. M. ARKIVOC 2010, 189.

[7] Reimann, S.; Danke, V.; Beiner, M.; Binder, W. H. J. Polym. Sci. , Part A: Polym. Chem. 2017, 55, 3736.

[8] Afon'kin, A. A.; Kostrikin, L. M.; Shumeiko, A. E.; Popov, A. F.; Matveev, A. A.; Matvienko, V. N.; Zabudkin, A. F. Russ. Chem. Bull. 2012, 61, 2149.

[9] Baldessari, A.; Mangone, C. P. J. Mol. Catal. B: Enzym. 2001, 11, 335.

[10] Sauer, D. R.; Kalvin, D.; Phelan, K. M. Org. Lett. 2003, 5, 4721

[11] Polshettiwar, V.; Varma, R. S. Tetrahedron Lett. 2008, 49, 2661.

[12] Sharghi, H.; Sarvari, M. H. Synth. Commun. 2003, 33, 207.

[13] Ganguly, N. C.; Roy, S.; Mondal, P. Tetrahedron Lett. 2012, 53, 1413.

[14] Allam, B. K.; Singh, K. N. Tetrahedron Lett. 2011, 52, 5851.

[15] Zhang, J.; Leitus, G.; Ben-David, Y.; Milstein, D. J. Am. Chem. Soc. 2005, 127, 12429 .

[16] Zhang, J.; Gandelman, M.; Shimon, L. J. W.; Rozenberg, H.; Milstein, D. Organometallics 2004, 23, 4026.

[17] Chan, W. K.; Ho, C. M.; Wong, M. K.; Che, C. M. J. Am. Chem. Soc. 2006, 128, 14796

[18] Hogberg, T.; Strom, P.; Ebner, M.; Ramsby, S. J. Org. Chem. 1987, 52, 2033.

[19] Arora, R.; Paul, S.; Gupta, R. Can. J. Chem. 2005, 83, 1137.

[20] Burkhardt, I.; Lauterbach, L.; Brock, N. L.; Dickschat, J. S. Org. Biomol. Chem. 2017, 15, 4432

[21] Lin, J. J.; Wu, J. J.; Ho, Y. S. J. Appl. Polym. Sci. 2001, 82, 435.

[22] Yang, K. W.; Cannon, J. G.; Rose, J. G. Tetrahedron Lett. 1970, 1791.

[23] Xie, L. Y.; Peng, S.; Liu, F.; Chen, G. R.; Xia, W.; Yu, X.; Li, W. F.; Cao, Z.; He, W. M. Org. Chem. Front. 2018, 5, 2604.

[24] Yu, X.; Zhou, F.; Chen, J.; Xiao, W. Acta Chim. Sinca 2017, 75, 86 (in Chinese).

(余晓叶, 周帆，陈加荣，肖文精，化学学报, 2017, 75, 86.)

[25] Zhang, J.; Niu, L.; Li, Y.; Liu, S.; Jiang, L. Chin. J. Org. Chem. 2018, 38, 1842 (in Chinese)

(张俊辉, 牛李智, 李映, 刘思, 姜林, 有机化学, 2018, 38, 1842.) 
[26] Greenlee, K. W.; Henne, A. L. Inorg. Synth. 1946, 2, 128.

[27] Willard, M. L.; Maresh, C. J. Am. Chem. Soc. 1940, 62, 1253.

[28] Kuzma, P. C.; Brown, L. E.; Harris, T. M. J. Org. Chem. 1984, 49, 2015.

[29] Philbrook, G. E. J. Org. Chem. 1954, 19, 623.

[30] Behun, J. D.; Levine, R. J. Am. Chem. Soc. 1959, 81, 5157.
[31] Ohmura, R.; Takahata, M.; Togo, H. Tetrahedron Lett. 2010, 51, 4378.

[32] Chinchilla, R.; Dodsworth, D. J.; Najera, C.; Soriano, J. M. Tetrahedron Lett. 2003, 44, 463.

[33] Ma, X. Y.; Lu, M. J. Chem. Res. 2011, 480.

(Zhao, C.) 